Overall improvement in the nationwide system of medical services has consequently boosted the number of successfully treated patients who suffer from head and neck cancer. It is essential to effectively prevent development of radiation-induced caries as the late effect of radiation therapy. Incidence and severity of radiationinduced changes within the teeth individually vary depending on the patient's age, actual radiation dose, size of radiation exposure field, patient's general condition and additional risk factors. Inadequately managed treatment of caries may lead to loss of teeth, as well as prove instrumental in tangibly diminishing individual quality of life in patients. Furthermore, the need to have the teeth deemed unyielding or unsuitable for the application of conservative methods of treatment duly extracted is fraught for a patient with an extra hazard of developing osteoradionecrosis (ORN), while also increasing all attendant therapeutic expenditures. The present paper aims to offer some practical insights into currently available methods of preventing likely development of radiation-induced caries.

Key words: radiotherapy, caries prevention, osteoradionecrosis, quality of life.

Contemp Oncol (Pozn) 2016; 20 (4): 287-290 DOl: $10.5114 / w 0.2015 .54081$

\section{Radiation-induced caries as the late effect of radiation therapy in the head and neck region}

\author{
Katarzyna Dobroś, Justyna Hajto-Bryk, Małgorzata Wróblewska, \\ Joanna Zarzecka
}

Department of Conservative Dentistry with Endodontics, Institute of Dentistry, Jagiellonian University Collegium Medicum, Krakow, Poland

\section{Introduction}

The incidence of malignant neoplasms in Poland in the past three decades has more than doubled, and, according to the prognosis put forward by Didkowska, by 2025 the number of new cases in both men and women will continue to grow $[1,2]$. At the same time, advances in medicine tangibly contributed to improving overall efficacy of early cancer detection, as reflected in a steadily increasing number of successfully treated patients. One of the widely applied methods of treatment of head and neck cancer consists in radiotherapy, be it used as a stand-alone, or a complementary method, following surgical intervention.

Advances in the techniques of irradiation, as well as the establishment of new regional oncological centres, facilitate treatment of an increasing number of patients, which has already resulted in the emergence of a brand-new category of patients. Following the radiotherapy treatment, the cancer patients are subject to constant monitoring by oncologists or other specialists. It is essential that not only the dentists, but also the attending physicians pay due attention to the overall condition of the oral cavity and dentition in this group of patients. The development of radiation-induced caries, also known as radiation caries, which is a late complication of radiotherapy, may even lead to a complete loss of teeth in patients who prior to the oncological treatment experienced no problems with their teeth whatsoever [3]. Missing teeth can greatly affect overall quality of life, so greater awareness of the likely complications that might occur in the oral cavity after irradiation treatment is absolutely vital.

Mucositis, hyposalivation, dysaesthesia, bacterial and fungal infections, osteoradionecrosis (ORN) and trismus are the most common complications of direct irradiation in patients suffering from head and neck cancer [4-11]. The incidence and severity of radiation-induced changes within the teeth individually vary, depending on the patient's age, actual radiation dose, size of radiation exposure field (position and actual size of the tumour), patient's general condition and additional risk factors, e.g. alcoholism, smoking, malnutrition [12]. During and after the radiotherapy some clinical symptoms may occur as an acute response to treatment. In the case of head and neck cancer radiotherapy, the most severe symptoms are those related to the acute mucosal reaction [13]. Radiation caries is not a primary effect of radiotherapy, but develops as a secondary condition. The main etiological factor in the development of caries is the bacteria encountered in the dental plaque. In the irradiated patients, however, it is the damage sustained by the serous cells of the salivary glands that is of essential importance, being the adverse side effect of the radiation therapy itself. As a result of the decreased amount of saliva flow and the changes in its properties, hyposalivation develops, which consequently impairs the self-cleaning of the teeth, with 
the resultant increased accumulation of dental plaque. The population of cariogenic bacteria, i.e. Lactobacillus and Streptococcus mutans, increases in the saliva, and so does the level of immunoglobulin A (IgAs). These changes may occur from the commencement of radiotherapy up to three months after its completion, and are believed to appreciably increase the risk of radiation caries [5]. Their immediate effect consists in a limited re-mineralisation of the enamel, combined with severe demineralisation at the same time [9].

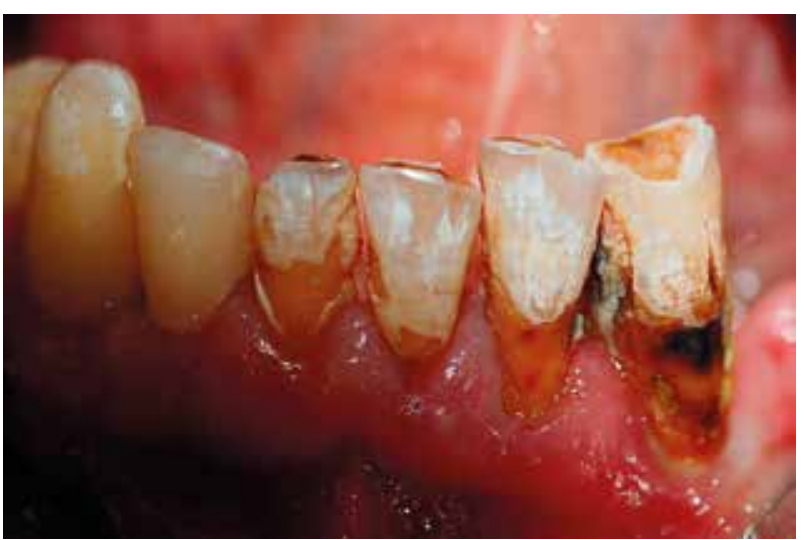

Fig. 1. Incisors with typical signs of radiation-induced caries (own research material)

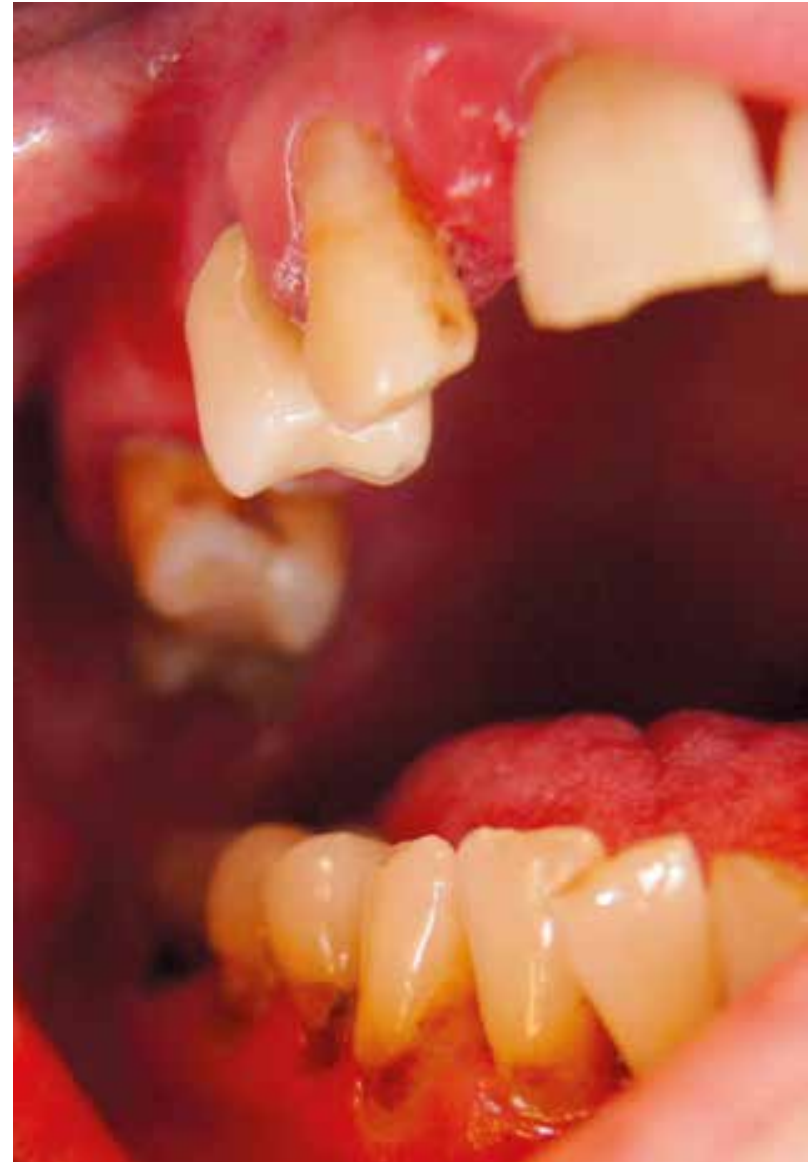

Fig. 2. Multiple caries affecting the cervical region of the teeth in an irradiated patient (own research material)
During and immediately after the radiotherapy, patients often report increased sensitivity to thermal and chemical stimuli (sweet, sour foods), which is associated with the lack of a protective layer of saliva and inflammation of the oral mucosa. Mucositis results in increased susceptibility to injury. Additional pain associated with tooth brushing often causes the patients to become negligent with regard to routine oral hygiene. Trismus and general malaise are also believed to be factors compromising oral hygiene [10, 14].

Radiation caries boasts a characteristic clinical course. It usually has a rapid onset and quick progress, which in the most extreme cases may lead to total destruction of originally healthy dentition within a year. Radiation caries is characterised by the absence of acute pain even in the advanced changes. Histologically, the changes encountered in radiation caries are of the same character as the ones resulting from other causes $[5,15]$. In clinical terms, radiation caries is located around the necks of the teeth, and typically affects more than one surface; it is therefore called caries circularis (Fig. 1). It is most characteristic that radiation caries usually sets in on the smooth surfaces of the incisors, premolars and molars, which as a rule are most resistant to decay (Fig. 2). This is due to the reduced secretion of saliva and consequently the much impaired self-cleaning of the teeth. The teeth located in the irradiated areas are discoloured, brownish, exhibit changes in translucency, and become brittle and prone to fracture (Figs. 3, 4) [5].

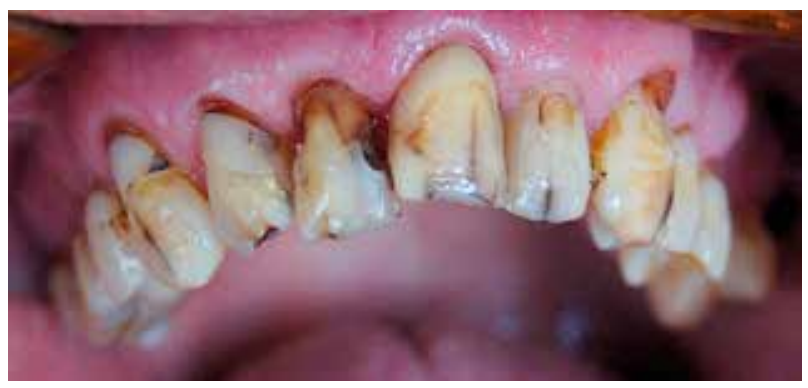

Fig. 3. Hard tissues of the teeth in an irradiated patient (own research material)

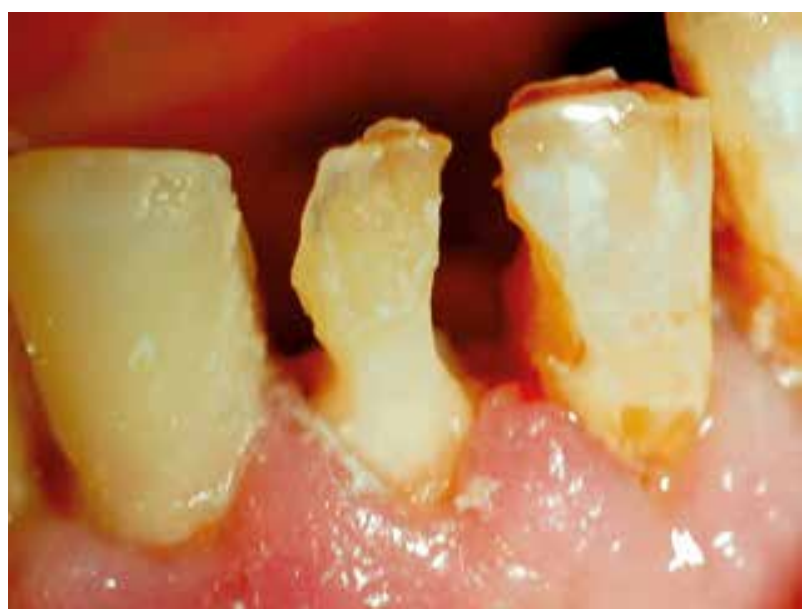

Fig. 4. Pathological fracture of the crown (own research material) 
The article presents the specifics of dental care for patients after radiotherapy, with particular emphasis on prevention and treatment of radiation-induced caries.

\section{Overview}

Thorough clinical dental examination combined with a detailed analysis of orthopantomograms prior to commencement of the radiation therapy allows one to effectively detect the potential focus of infection, and plan optimum pre-treatment of the oral cavity. This appreciably reduces the risk of developing radiation caries, which may potentially lead to total destruction of dentition and, consequently, the need for extraction. Fostering close co-operation between the oncologist and the dentist is therefore deemed essential, especially at an early stage of preparing a patient for radiotherapy. Pre-treatment of the oral cavity, particularly any dental extractions due, should be completed two weeks before the scheduled commencement of radiotherapy $[5,12,16]$.

With a view to reducing the likelihood of developing radiation caries, the patients are strongly advised to implement a preventive fluoride regimen before the radiotherapy commences. For this purpose, fluoride gels ( $1 \% \mathrm{NaF})$ are to be used at least every other day, or toothpaste with an increased fluoride content (3.0\% NaF) twice a day, mouth rinses containing fluoride $(0.05 \% \mathrm{NaF})$, or ones containing chlorhexidine as an adjunct (to be used for a maximum of 2-3 weeks) [12]. During the conservative treatment in this group of patients amalgam fillings should be avoided, as they become a source of secondary radiation [4]. Any treatment of the oral cavity, while the radiotherapy is already in progress, is of a symptomatic character only and applies in particular to ailments associated with mucositis, candidiasis, xerostomia, and opportunistic bacterial and viral infections. Due to these conditions and weight loss during radiotherapy, the patients remain on a soft food diet rich in carbohydrates, thereby further aiding the development of caries $[6,13]$. It is for this very reason that oral hygiene instructions combined with a preventive fluoride regimen are vital, and so is the dietary advice for the patients.

After the radiotherapy treatment has been concluded, it is essential to further motivate the patient to maintain adequate oral hygiene. Regular visits should be conducted every three months, as those are aimed at professional prophylaxis, symptomatic treatment of any ailments, as well as at the detection and treatment of any new cavities. Unfortunately, there are no specific guidelines for clinical management of radiation caries in this group of patients, so the actual treatment practices are based on the individual clinical experience of the attending dental surgeons rather than on any rigidly formulated regimen protocols. Galetti et al. recommend the use of composite materials and resin-modified glass ionomer cements, since no difference in the strength of binding to dentin was proven with regard to the adhesive materials in the irradiated teeth, in comparison with the control group [4]. The observations of Hu et al. indicated that glass ionomer cements could be used in the treatment of radiation-induced, root surface carious lesions with good results [14].
If left untreated, caries leads to the development of pulp disease and periapical tissue disease, thereby necessitating endodontic treatment. Failure to carry out root canal treatment results in the necessity to have the inflamed teeth extracted, which in this group of patients is associated with an increased risk of osteoradionecrosis (ORN) [1618]. Should it prove necessary to have the teeth extracted, any such extraction procedures should be carried out by specialists, effectively ensuring minimum trauma in the process, in conjunction with subsequent follow-up and adjuvant hyperbaric oxygenation [17]. The above notwithstanding, there is still ORN risk that also needs to be addressed. The incidence of ORN is associated with ionizing radiation, which irreversibly damages the cells and vascular elements of the tissue, resulting in difficulties in healing of the post-extraction wound [16]. Unfortunately, many dentists opt for excessively premature tooth extractions, although with quiet acquiescence of their patients. It is estimated that post-extraction ORN is encountered in 2-18\% of patients who have previously undergone radiotherapy [18]. Patients may experience pain, trismus and purulent exudate from the necrotic area [19]. Treatment of ORN is rather long and drawn-out, and it can take up to 6 months, so before the decision of tooth extraction is ultimately made, the patients should be adequately advised about potential complications such as ORN. It should also be borne in mind that incomplete dentition should be remedied either by prosthodontic or implantology treatment. In irradiated patients the risk of implant failure is higher than in patients in good general health [20-22]. Therefore the option of endodontic treatment should always be considered in each case, with a view to effectively helping the patient to retain his own teeth.

\section{Summary}

Evaluation of the quality of life in patients successfully treated for cancers of the head and neck includes their ability to chew in order to ensure proper nutrition. This is facilitated by the retained dentition. This in turn may be achieved by enhancing the actual awareness of this issue in both the oncologists and the dentists, as they attend to and manage prophylaxis and treatment of radiation-induced dental caries. With a view to minimising the side effects of head and neck radiotherapy, fostering close co-operation between the oncologist and the dentist at an early stage of preparing a patient for radiotherapy is therefore deemed essential.

The authors declare no conflict of interest.

\section{References}

1. Wojciechowska U, Didkowska J, Zatoński W. Malignant tumors in Poland in 2010. 2012;.

2. Didkowska J, Wojciechowska U, Zatoński W. Prognosis of morbidity and mortality of cancer in Poland until 2025. 2009.

3. Cooper JS, Fu K, Marks J, Silverman S. Late effects of radiation therapy in the head and neck region. Int J Radiat Oncol Biol Phys 1995; 31: 1141-64. 
4. Galetti R, Santos-Silva AR, Nogueira da Gama Antunes A, de Abreu Alves F, Lopes MA, de Goes MF. Radiotherapy does not impair dentin adhesive properties in head and neck cancer patients. Clin Oral Investig 2014; 18: 1771-8.

5. Kielbassa AM, Hinkelbein W, Hellwig E, Meyer-Luckel H. Radiation-related damage to dentition. Lancet Oncol 2006; 7: 326-35.

6. Lieshout HF, Bots CP. The effect of radiotherapy on dental hard tissue-a systematic review. Clin Oral Investig 2014; 18: 17-24.

7. Schiodt M, Hermund NU. Management of oral disease prior to radiation therapy. Support Care Cancer 2002; 10: 40-3.

8. Bańkowski K, Ciesielski P, Łaszkiewicz J. Complications from ora cavity after radiotherapy head and neck neoplasm. Por Stomatol 2005; 3: 22-6.

9. Sieńko E. Oral complications in patients after radiotherapy for head and neck cancers. Onkol Pol 2007; 10: 130-2.

10. Stryjski A, Borysewicz-Lewicka M. Patients oral health status after head and neck neoplasm radiotherapy. Dent Med Probl 2007; 44: 177-83.

11. Olczak-Kowalczyk D, Daszkiewicz M, Kowalczyk W, Daszkiewicz P, Bagińska-Dembowska B, Perek D. Selected dental problems in patients exposed to anticancer treatment based on CTCAEv3.0. Part II. Stomat Współcz 2007; 14: 30-7.

12. Vissink A, Burlage FR, Spijkervet FK, Jansma J, Coppes RP. Prevention and treatment of the consequences of head and neck radio therapy. Crit Rev Oral Biol Med 2003; 14:2 13-25.

13. Wygoda A, Składowski K, Sąsiadek W, Hutnik M. Acute radiation mucosal reaction in patients with head and neck cancer. Contemp Oncol (Pozn) 2007; 11: 210-9.

14. Hu JY, Chen XC, Li YQ, Smales RJ, Yip KH. Radiation-induced root surface caries restored with glass-ionomer cement placed in conventional and ART cavity preparations: Results at two years. Aust Dent J 2005; 50: 186-90.

15. Silva AR, Alves FA, Berger SB, Giannini M, Goes MF, Lopes MA. Radiation-related caries and early restoration failure in head and neck cancer patients. A polarized light microscopy and scanning electron microscopy study. Support Care Cancer 2010; 18: 83-7.

16. Koga DH, Salvajoli JV, Kowalski LP, Nishimoto IN, Alves FA. Dental extractions related to head and neck radiotherapy: Ten-year experience of a single institution. Oral Surg Oral Med Oral Pathol Oral Radiol Endod 2008; 105: e1-6.

17. Koga DH, Salvajoli JV, Alves FA. Dental extractions and radiotherapy in head and neck oncology: Review of the literature. Oral Dis 2008; 14: 40-4

18. Nabil S, Samman N. Incidence and prevention of osteoradionecrosis after dental extraction in irradiated patients: A systematic review. Int J Oral Maxillofac Surg 2011; 40: 229-43.

19. Oh HK, Chambers MS, Martin JW, Lim HJ, Park HJ. Osteoradionecrosis of the mandible: Treatment outcomes and factors influencing the progress of osteoradionecrosis. J Oral Maxillofac Surg 2009; 67: 1378-86.

20. Zheng M, Li L, Tang Y, Liang X. How to improve the survival rate of implants after radiotherapy for head and neck cancer? J Periodontal Implant Sci 2014; 44: 2-7.

21. Chen H, Liu N, Xu X, Qu X, Lu E. Smoking, radiotherapy, diabetes and osteoporosis as risk factors for dental implant failure: A Meta-Analysis. PLoS ONE 2013; 8: e71955.

22. Chambrone L, Mandia J Jr, Shibli JA, Romito GA, Abrahao M. Dental implants installed in irradiated jaws: a systematic review. J Dent Res 2013; 92 (12 Suppl): 119S-30S.

\section{Address for correspondence}

\section{Katarzyna Dobroś}

Department of Conservative Dentistry with Endodontics Institute of Dentistry

Jagiellonian University Collegium Medicum

Montelupich 4

31-155 Krakow, Poland

e-mail: katarzyna.dobros@uj.edu.pl

Submitted: 12.11 .2014

Accepted: $\quad 7.04 .2015$ 